\title{
DETERMINING THE EFFECTIVENESS OF THE DIFFERENT PREPARATION PROTOCOLS FOR PLATELET-RICH PLASMA (PRP) IN YIELDING HIGHER CONCENTRATIONS OF PLATELETS
}

\author{
Kavitha G, Sangeetha VN, Shani S, Murali MR, Raja EA, Rukmanikanthan S and David C \\ Tissue Engineering Group, Department of Orthopaedic Surgery, NOCERAL, \\ Faculty of Medicine, University of Malaya, Kuala Lumpur.

\section{Correspondence:} \\ Sangeetha Vasudevaraj Naveen \\ Tissue Engineering Group (TEG), National Orthopaedic Centre of Excellence in Research and Learning (NOCERAL), \\ Department of Orthopaedic, Faculty of Medicine, \\ University of Malaya, \\ 50603 Lembah Pantai, \\ Kuala Lumpur, Malaysia. \\ Tel: +603-7967 7543, +6012-532 7289, Fax: +603-7949 4642 \\ E-mail: sangeethanaveen@um.edu.my
}

\begin{abstract}
INTRODUCTION:

Despite the various methods described in producing platelet-rich plasma (PRP), it is well established that this biological product in its many preparations have been proven to enhance wound healing. However, very little have been known about the efficacy of these methods hence there is a lack of evidence in the superiority of one method over another. Thus, a study was conducted to compare these different protocols to determine which produces the highest concentration of platelets.
\end{abstract}

\section{METHODS:}

Peripheral blood was obtained from 24 healthy volunteers. Four different protocols using similar 2 step centrifugation methods of preparing PRP were applied to an equal number of samples in this study. Platelet counts were performed on whole blood (without processing), PRP preparations and platelet-poor plasma (PPP).

\section{RESULTS:}

All protocols produced higher amounts of platelet concentrates in PRP preparations than plasma. However, centrifugation at $150 \mathrm{~g}$ for 10 minutes followed by another at $450 \mathrm{~g}$ at 10 minutes produces significantly higher amount of platelets concentration $(p<0.05)$.

\section{CONCLUSION:}

Optimizing the protocols to produce PRP appears to be important in obtaining a maximal yield of platelet concentrate. Here the protocol described has shown to provide significant concentration yield over all others.

Keywords: platelet-rich-plasma, growth factors, centrifugal forces

\section{Introduction}

Platelet rich plasma (PRP) is a highly concentrated form of autogenous platelets, providing a rich and readily obtainable source of a diverse group of growth factors(1). Its importance is related to the large variety of growth factors involved in healing that are physiologically contained in platelet $\alpha$-granules (2). Although the role of platelet concentrates in treating various haematological conditions and diseases have been well established, their roles in wound healing have only been recently described.
PRP mainly consist of platelets and several growth factors which includes (but not limited to) platelet-derived growth factor (PDGF), transforming growth factor (TGF), vascular endothelial growth factor (VEGF), endothelial growth factor (EGF) and insulin-like growth factor (IGF). These growth factors are released from activated platelet to initiate and modulate wound healing in both soft and hard tissues. Growth factors found in higher concentrations are thought to help in accelerating and enhance wound healing through tissue repair mechanisms such as chemotaxis, 
cell proliferation, angiogenesis, extracellular matrix deposition and remodelling $(3,4)$. PRP which contains high concentrations of growth factors, is used for various applications mainly for wound healing that may shorten the healing period. Besides, PRP are widely used in oral, maxillofacial surgery, tendon repairs, muscle therapy and many more.

Based on our literature review, there are several ways to prepare platelet concentrate (PRP), all of which promising higher platelet concentrations. However, we have found there has been no reference to a study which makes a side to side comparison analyses on these various methods and thus provide no evidence of the effectiveness of one particular method over the other. Thus, a study was conducted to compare the different methods of preparing PRP and to determine which method best produces the highest platelet concentrate. In addition, this study will also reconfirm the reliability of different preparation of PRP as claimed in the different literatures.

\section{Methods}

PRP is prepared via a 2-step centrifugation procedure using venous blood. The centrifuge speed, the amount of blood collected, the type of equipment used for collection of blood differs according to the type of preparations described by the various authors. Blood was obtained from 24 subjects who have no previous known medical illness and appeared well on the day where the procedure was performed.

A venepuncture was performed by a phlebotomist to obtain the peripheral blood. $10 \mathrm{ml}$ of blood was aspirated with a $21 \mathrm{G}$ needle and was mixed with $18 \mathrm{mg}$ of anticoagulant K2 potassium salt of ethyleneediaminetetraacetic acid (EDTA) to avoid coagulation. $0.2 \mathrm{ml}$ of blood was taken and placed in a sterile tube where whole blood count was performed. PRP was isolated from whole blood at different centrifugal force at different time period which results in a 0.5-1 $\mathrm{ml}$ "buffy-coat" extract. The layer was then separated centrifuged again to obtain a two-part plasma: the upper layer which is the poor-platelet plasma (PPP) and the lower layer known as PRP. The PRP and PPP were then separated and place in sterile tubes. A series of platelet count was performed using the Sysmex XT-1800 machine.

\section{Statistical Analysis}

The data obtained were analyzed using statistical software (SPSS for Windows; PASW Statistics version 18). The number of platelets in whole blood, the number of platelets in PRP and the number of platelets in PPP were compared with different methods of preparations. Non-parametric test $(p<0.05)$ were employed in this study.

Table 1: The different centrifugation speed, period of time and temperature taken for the comparison of which best produces the highest platelet counts.

\begin{tabular}{|c|c|c|c|c|c|c|}
\hline Method & $\begin{array}{r}1^{\text {st }} \text { Centrifugation } \\
\text { speed }\end{array}$ & $\begin{array}{r}\text { Time } \\
\text { (Minutes) }\end{array}$ & Temperature $\left({ }^{\circ} \mathrm{C}\right)$ & $\begin{array}{r}2^{\text {nd }} \text { Centrifugation } \\
\text { Speed }\end{array}$ & $\begin{array}{r}\text { Time } \\
\text { (minutes) }\end{array}$ & Temperature $\left({ }^{\circ} \mathrm{C}\right)$ \\
\hline 1 & $2000 \mathrm{~g}$ & 30 & 20 & $250 \mathrm{~g}$ & 20 & 20 \\
\hline 2 & $150 \mathrm{~g}$ & 10 & 20 & 450 & 10 & 20 \\
\hline 3 & $215 \mathrm{~g}$ & 10 & 20 & 865 & 10 & 20 \\
\hline 4 & 1125 & 10 & 20 & 300 & 25 & 20 \\
\hline
\end{tabular}

\section{Isolation of blood through venipuncture.}

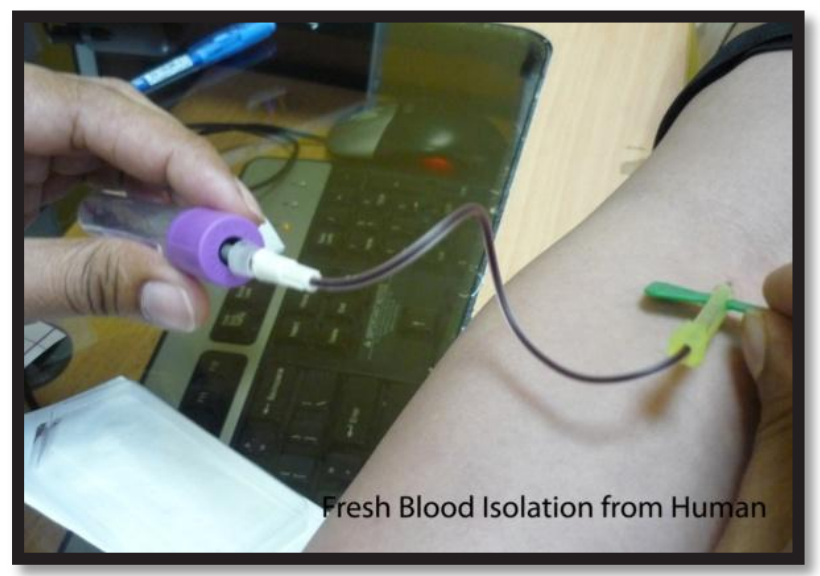


Obtained blood was transferred into the $10 \mathrm{ml}$ EDTA tube (anti-coagulation) and centrifuged at different speed as stated in Table 1
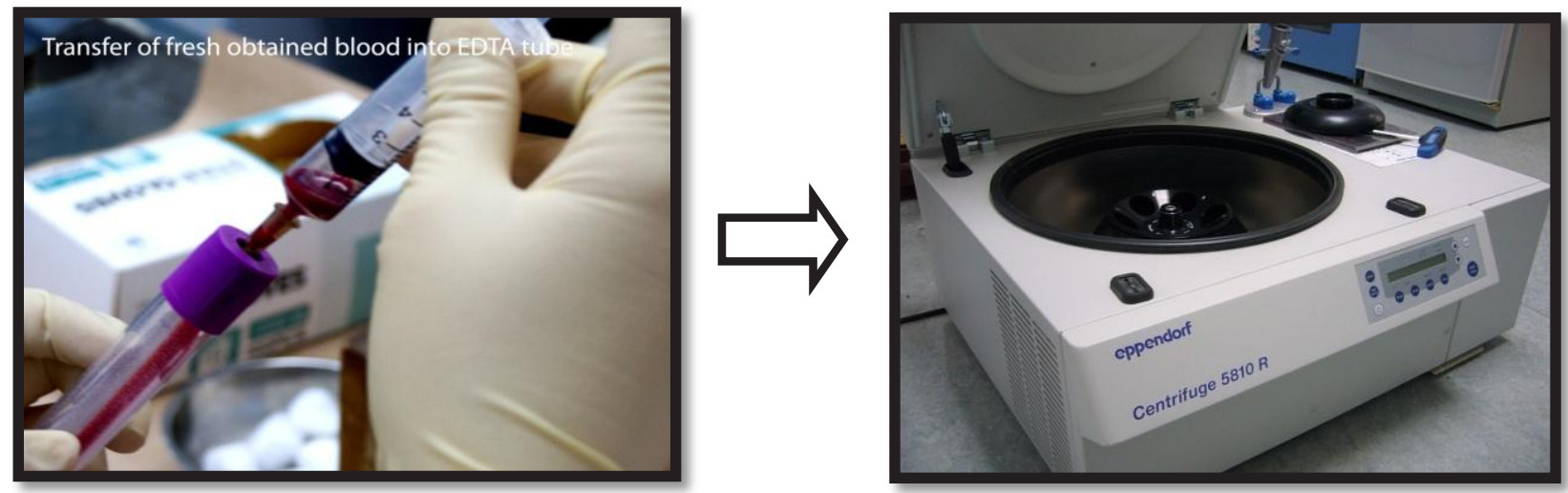

Three layers were formed (Top layer: Plasma, Middle layer: Buffy Coat, Lowest Layer: Red Blood Cells). 1 $\mathrm{ml}$ of plasma layer right above the Buffy coat was obtained and centrifuged again.
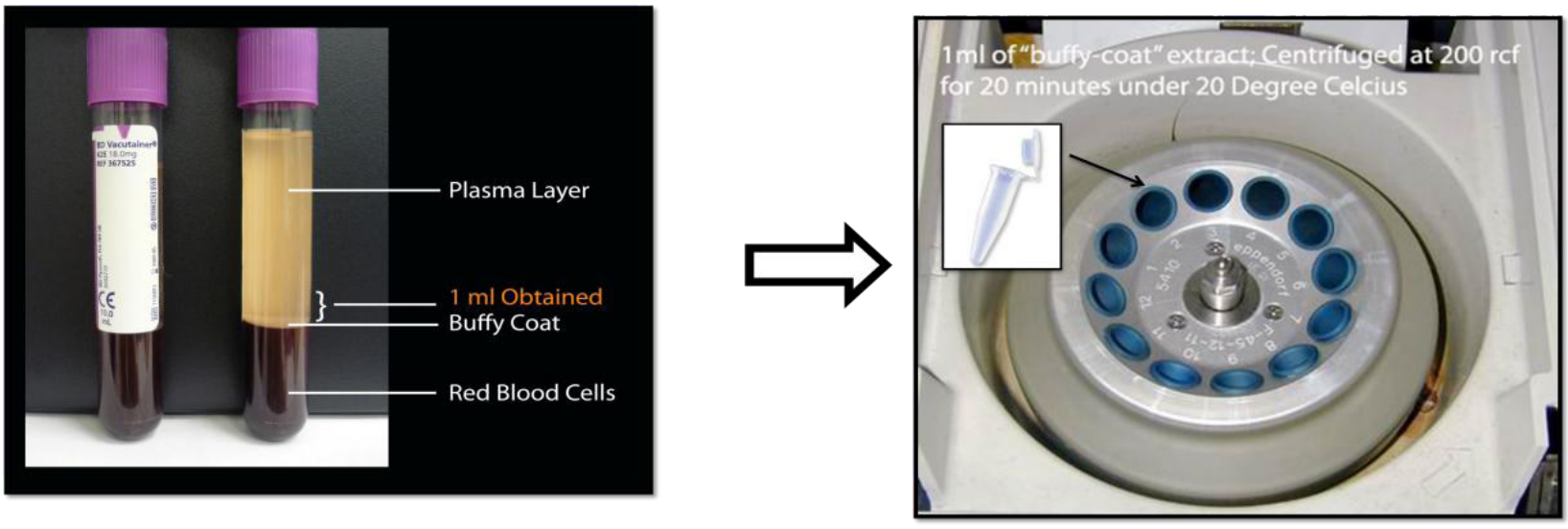

Two layers were formed: Lower layer Platelet-Poor Plasma (PPP) and Upper Layer of Platelet-Rich Plasma (PRP)
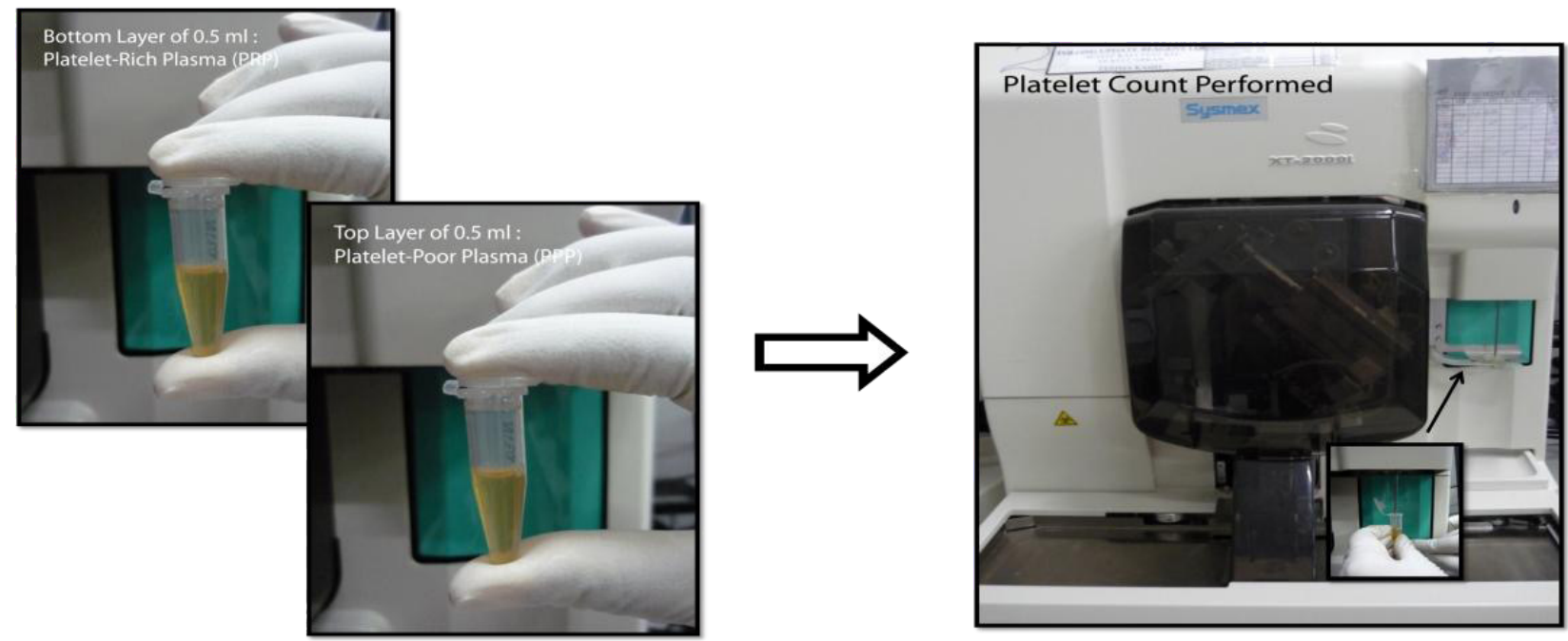

Flow Chart 1: Shows the two centrifugation technique that was performed. Data was attained for whole blood counts, PRP counts and PPP counts. 


\section{Results}

PRP was prepared using different centrifugal forces which were described by previous authors (5-7). Regardless of the method used, the numbers of platelets in PRP were of higher value as compared to the number of platelets in whole blood. Figure 1 to 4 is a summary comparing the values attained based on platelet counts from whole blood and PRP. All four methods proved that platelet counts for PRP is of 2-5 folds higher as compared to the platelet counts in whole blood.

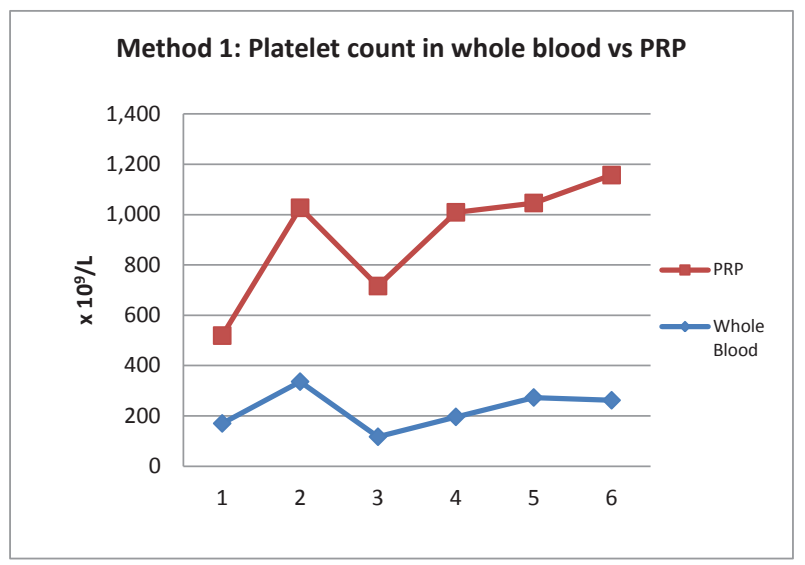

Figure 1: The graph above shows the platelet count in whole blood and PRP obtained by using Method 1

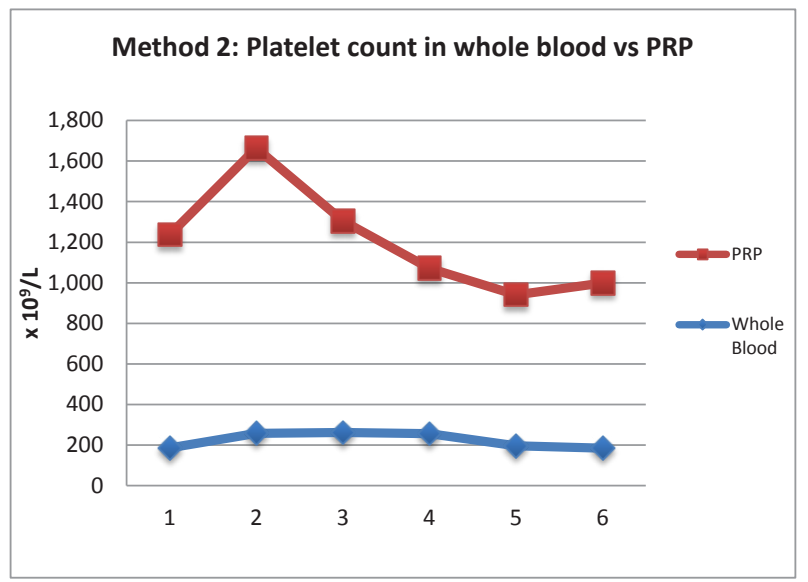

Figure 2: The graph above shows the platelet count in whole blood and PRP obtained by using Method 2

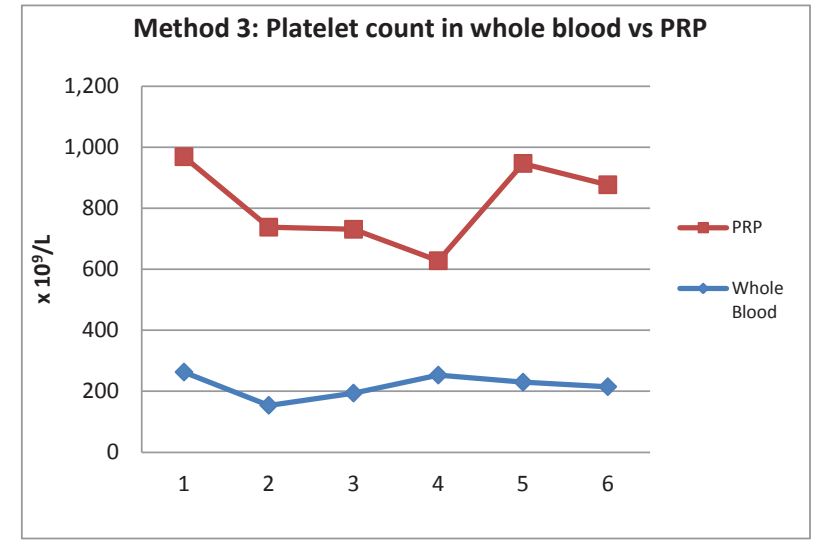

Figure 3: The graph above shows the platelet count in whole blood and PRP obtained by using Method 3

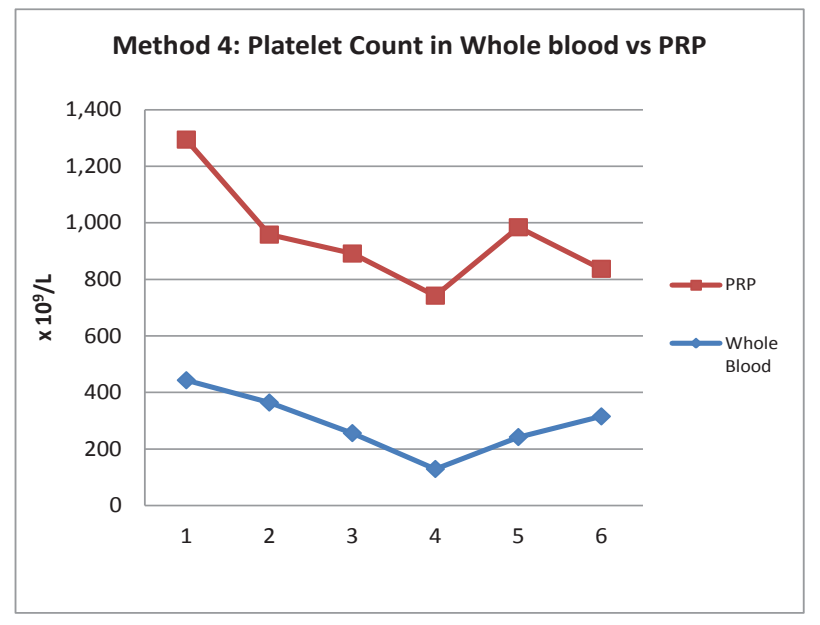

Figure 4: The graph above shows the platelet count in whole blood and PRP obtained by using Method 4

There were 11 females and 13 male donors in this study. The mean of the age for the subjects who participated for this study lies within the range of 24 to 29. There were no significant differences between the mean age and the values attained for both sexes (Table 2 and 3). Based on statistical analysis, it was found that the distribution of platelet counts in whole blood in method 1,2,3 and 4 were not significantly different $(p<0.05)$. It was also found that that the distribution of platelets in PRP attained using methods 1, 2, 3 and 4 were of normal distribution.

Table 2: Mean Values for Platelets in Whole Blood, PRP and PPP.

\begin{tabular}{rrrrrrrr}
\hline \multicolumn{2}{c}{ Number of Subjects } & \multicolumn{4}{c}{ Mean no of Platelets (x 109/L) } \\
\hline Method & Males & Female & Age & WB & PRP & PPP & p-value \\
\hline 1 & 4 & 2 & 28.83 & 256.67 & 686.67 & 24.50 & 0.135 \\
2 & 2 & 4 & 24.67 & 223.83 & 979.17 & 59.17 & 0.02 \\
3 & 3 & 3 & 24.83 & 218.17 & 596.83 & 35.00 & 0.135 \\
4 & 4 & 2 & 24.50 & 291.50 & 659.50 & 212.60 & 0.007 \\
\hline
\end{tabular}

* $p$-value denoted is by comparing $p$-values of PRP and PPP by using Mann-Whitney $\mathrm{U}$ test. 
It is found that the mean number of platelet counts for PRP obtained by following method 2 produces the highest platelet count (Kruskall-wallis: $\mathrm{p}<0.05$ ). All other methods only produced between 2.2 to 2.75 fold increase in platelet counts (table 3).

Table 3: Data analysed on the number of folds comparing whole blood with PRP.

\begin{tabular}{rrrrr}
\hline \multicolumn{6}{c}{ Mean no of Platelets (x 109/L) } \\
\hline Methods & WB & PRP & $\begin{array}{r}\text { Number of } \\
\text { folds }\end{array}$ & p-value \\
& & & 2.67 & \\
2 & 256.67 & 686.67 & 4.37 & 0.87 \\
3 & 223.83 & 979.17 & 2.74 & \\
4 & 218.17 & 596.83 & 2.26 & \\
\hline
\end{tabular}

${ }^{*}$ p-value denoted is by comparing $p$-values of PRP with different methods by using post hoc - Tamhane's T2 test.

\section{Discussion}

The realization in the vast potential of PRP in clinical applications have made the use of this simple to produce biological product for maxillofacial surgery, foot and ankle surgery (8), osteal defects repair $(9,10)$, muscle and tendon therapy (11-13) and many more, a dispensible alternative to current more elegant and expensive methods of treatment including stem cell therapies. However, before the use of PRP can be effective, several issues needs to be addressed which includes identifying the most important method to produce the most effective therapeutic concentration possible. The type of anti-coagulant, centrifugal speeds, the amount and the type of growth factors existing in PRP, the number of platelets in the donor's blood and PRP itself as well as the clinical applications of PRP all play an important role in determining the maximal yield of platelet concentrate and therefore must be carefully considered before being applied into clinical applications (14-18). To our knowledge, we found that there were many methods to preparing PRP but no similar comparative study as to the one presented in the present report have been described previously. This study demonstrates the importance of determining optimization methods and standard practices which will eventually lead to the best outcome possible of PRP products.

Further determining the highest possible concentration using various methods of preparations, it is also noteworthy to determine the concentrations of platelets required for wound healing or other applications to be succesful. Haynesworth et al demonstrated that the proliferation of adult mesenchymal stem cells and their differentiation were directly related to the platelet concentration. They showed a dose-response curve, which indicated that, to produce a sufficient cellular response to platelet concentrations, concentration of approximately 4 to 5 fold increase of platelet count as compared to baseline platelet count would be required. It was therefore important to note, that if this study had made its way into clinical applications, method 2 would have been the only method which would have produced a significant clinical outcome while the other methods would prove to be sub-optimal. As most individuals have a baseline platelet count of $200,000 \pm 75,000 / \mu \mathrm{L}$, a PRP platelet count of 1 million/ $\mu \mathrm{L}$ as measured in the standard 6-ml aliquot has become the benchmark for "therapeutic PRP".

Based on the mean platelet counts, the highest in mean number of platelets concentration in PRP was produced by applying method 2 which was $979.17 \times 10^{9} / \mathrm{L}$. The highest mean number of platelet in PRP is followed by method 1,4 and 3 (in a descending order) which falls within the range of 500 to $700 \times 10^{9} / \mathrm{L}$. In reference to the study conducted by Marx et al, other than method 2, all other methods would not be beneficial for patients receiving ineffective concentration of PRP. Due to this factor, FDA cleared devices (concentrates platelets) must consistently achieve this therapeutic levels of platelet concentration and thus growth factor release, in order to be registered as a therapeutic device $(17,18)$.

As the objective of this study was to compare the different techniques in order to attain the best or the highest platelet count in PRP, the changing variable is the centrifugal forces. Method 1 and method 4 uses "hard spin" for the first centrifugation and "soft spin" for the second centrifugation. Whereas, Method 2 and 3 starts off with soft spin for the first centrifugation and then followed by hard spin. Based on the values attained in this study, it was found that by applying method 2 produces the highest mean number of platelets in PRP, it can be said that, in order to obtain highest number of platelet concentrate, soft spin can be a better option. But this contradicts as Method 1, whereby we used hard spin and then followed by soft spin and this produces highest mean number of platelets as compared to method 3 and 4 . There are several articles published that uses hard spin and then soft spin for the second centrifugation and documented success rate in obtaining PRP with high number of platelets $(3,19,20)$. Thus, it is unclear and undefined whether the hard spin or the soft spin as the centrifugation force or vice versa for the second centrifugation force as far as obtaining highest number of platelets in PRP is concerned. Also, further studies should be conducted looking into the platelet function based on these using soft spin and hard spin as well as looking into these platelet function in terms of in vivo trials.

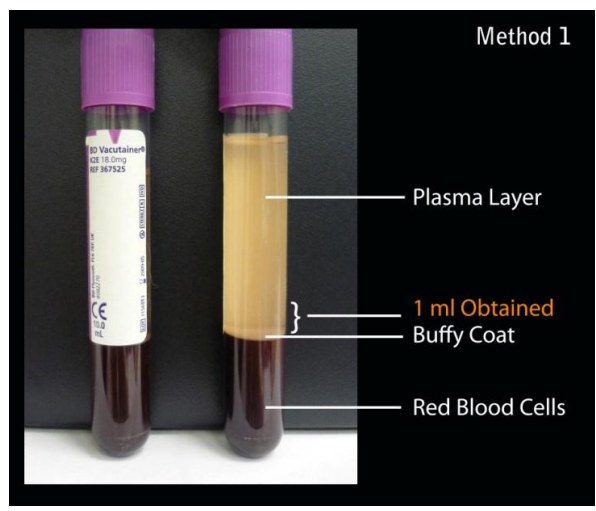

Figure 5: Images taken after the first centrifugation using method 1 
Several limitations were identified in this study and were carefully addressed where possible. One of which was the difficulty in distinguishing the plasma layer in our PRP preparations. Based on the images above, it was found that method number 1 shows a clear layer of plasma, buffy coat as well as red blood cells. However, in other methods, it was found to be difficult to delineate the exact PRP layer which lies directly above the buffy coat $(21,22)$. Furthermore, when it comes to the preparation of platelet, it was difficult to isolate the plasma layer right above the buffy coat running the risk of contaminating the PRP with red blood cells. It therefore appears that the method of sampling PRP in all methods is more of an art rather than pure science and therefore a more amicable and standardized approach to extract the exact layer of platelet concentration must be developed if a more replicable study is to be produced. In addition, although method 2 produced the highest mean number of platelets in PRP, the number of functional platelets remains an unanswered question. Thus, further studies to test the function of PRP in in vivo trials must be considered.

\section{Conclusion}

The proposed protocol that is used in method 2 which involved centrifugation at $150 \mathrm{~g}$ for 10 minutes followed by another at 450g at 10 minutes has shown to produce the highest mean number of platelets concentrations in PRP.

\section{Acknowledgement}

Authors are thankful to E-Science grant (12-02-03-2087) for the financial support.

\section{References}

1. Butterfield KJ, Bennett J, Gronowicz G, Adams D. Effect of platelet-rich plasma with autogenous bone graft for maxillary sinus augmentation in a rabbit model. Journal of Oral and Maxillofacial Surgery. 2005;63(3):370-6.

2. Dugrillon A, Eichler H, Kern S, Kluter H. Autologous concentrated platelet-rich plasma (cPRP) for local application in bone regeneration. Int J Oral Maxillofac Surg. 2002 Dec;31(6):615-9.

3. Marx RE, Carlson ER, Eichstaedt RM, Schimmele SR, Strauss JE, Georgeff KR. Platelet-rich plasma : Growth factor enhancement for bone grafts. Oral Surgery, Oral Medicine, Oral Pathology, Oral Radiology, and Endodontology. 1998;85(6):638-46.

4. Sammartino G, Tia M, Marenzi G, Espedito di Lauro A, D’Agostino E, Claudio PP. Use of Autologous PlateletRich Plasma (PRP) in Periodontal Defect Treatment After Extraction of Impacted Mandibular Third Molars. Journal of Oral and Maxillofacial Surgery. 2005;63(6):766-70.

5. Högman CF, Berséus O, Eriksson L, Gulliksson H. Buffy-coat-derived platelet concentrates: Swedish experience. Transfusion Science. 1997;18(1):3-13.
6. Mooren RECM, Merkx MAW, Bronkhorst EM, Jansen JA, Stoelinga PJW. The effect of platelet-rich plasma on early and late bone healing: an experimental study in goats. International Journal of Oral and Maxillofacial Surgery. 2007;36(7):626-31.

7. Freymiller EG, Aghaloo TL. Platelet-rich plasma: ready or not? Journal of Oral and Maxillofacial Surgery. 2004;62(4):484-8.

8. Gandhi A, Bibbo C, Pinzur M, Lin SS. The Role of Platelet-Rich Plasma in Foot and Ankle Surgery. Foot and Ankle Clinics of North America. 2005;10(4):621-37.

9. Demir B, Sengun D, Berberoglu A. Clinical evaluation of platelet-rich plasma and bioactive glass in the treatment of intra-bony defects. J Clin Periodontol. 2007 Aug;34(8):709-15.

10. Kasten P, Vogel J, Geiger F, Niemeyer P, Luginbühl R, Szalay K. The effect of platelet-rich plasma on healing in critical-size long-bone defects. Biomaterials. 2008;29(29):3983-92.

11. de Mos $M$, van der Windt AE, Jahr $H$, van Schie HTM, Weinans $\mathrm{H}$, Verhaar JAN, et al. Can Platelet-Rich Plasma Enhance Tendon Repair? The American Journal of Sports Medicine. 2008 June 2008;36(6):1171-8.

12. Mishra A, Pavelko T. Treatment of Chronic Elbow Tendinosis With Buffered Platelet-Rich Plasma. The American Journal of Sports Medicine. 2006 November 2006;34(11):1774-8.

13. Mishra A, Woodall Jr J, Vieira A. Treatment of Tendon and Muscle Using Platelet-Rich Plasma. Clinics in Sports Medicine. 2009;28(1):113-25.

14. Landesberg R, Roy M, Glickman RS. Quantification of growth factor levels using a simplified method of platelet-rich plasma gel preparation. Journal of Oral and Maxillofacial Surgery. 2000;58(3):297-300.

15. Landesberg R, Moses M, Karpatkin M. Risks of using platelet rich plasma gel. Journal of Oral and Maxillofacial Surgery. 1998;56(9):1116-7.

16. Landesberg R. Controlled Delivery of Growth Factors Derived From Platelet-Rich Plasma. Journal of Oral and Maxillofacial Surgery. 2006;64(9, Supplement 1):87-.

17. Marx RE. Platelet-rich plasma: evidence to support its use. Journal of Oral and Maxillofacial Surgery. 2004;62(4):489-96.

18. Marx RED. Platelet-Rich Plasma (PRP): What Is PRP and What Is Not PRP? [Article]. 2001;10(4):225-8.

19. Kanno T, Takahashi T, Tsujisawa T, Ariyoshi W, Nishihara T. Platelet-rich plasma enhances human osteoblastlike cell proliferation and differentiation. Journal of Oral and Maxillofacial Surgery. 2005;63(3):362-9.

20. Mrowiec ZR, Gelbart T, Oleksowicz L, Dutcher JP, De Leon-Fernandez $M$, Lalezari $P$, et al. A Novel Technique for Preparing Improved Buffy Coat Platelet Concentrates. Blood Cells, Molecules, and Diseases. 1995;21(1):25-33.

21. Gotcher JJE. Platelet Rich Plasma (PRP). Journal of Oral and Maxillofacial Surgery. 2005;63(8, Supplement 1):15-.

22. Griffin XL, Smith CM, Costa ML. The clinical use of platelet-rich plasma in the promotion of bone healing: A systematic review. Injury. 2009;40(2):158-62. 